\title{
Wigner-Araki-Yanase theorem on Distinguishability
}

\author{
Takayuki Miyadera * and Hideki Imai *,† \\ * Research Center for Information Security (RCIS), \\ National Institute of Advanced Industrial Science and Technology (AIST). \\ Daibiru building 1102, Sotokanda, Chiyoda-ku, Tokyo, 101-0021, Japan. \\ (e-mail: miyadera-takayuki@aist.go.jp) \\ † Graduate School of Science and Engineering Course of Electrical, Electronic, and Communication Engineering, \\ Chuo University. \\ 1-13-27 Kasuga, Bunkyo-ku, Tokyo 112-8551, Japan .
}

\begin{abstract}
The presence of an additive conserved quantity imposes a limitation on the measurement process. According to the Wigner-Araki-Yanase theorem, the perfect repeatability and the distinguishability on the apparatus cannot be attained simultaneously. Instead of the repeatability, in this paper, the distinguishability on both systems is examined. We derive a trade-off inequality between the distinguishability of the final states on the system and the one on the apparatus. The inequality shows that the perfect distinguishability of both systems cannot be attained simultaneously.
\end{abstract}

PACS numbers: 03.65.Ta, 03.67.-a

According to the Wigner-Araki-Yanase theorem, the presence of an additive conserved quantity imposes a limitation on the measurement process. Wigner, and later Araki and Yanase, showed [1-3] that in the sense of von Neumann's ideal measurement one cannot precisely measure observables which do not commute with the conserved quantity. That is, the repeatability of the measurements and the perfect distinguishability of the final states on the measuring apparatus cannot be realized simultaneously. On the other hand, if we abandon the repeatability condition, the perfect distinguishability of the final states on the apparatus can be attained [4,5]. Ozawa [6,7] has derived a quantitative relation between the noise operator and the disturbance operator by Robertson type inequality to discuss the trade-off between the repeatability and the distinguishability on the apparatus. We, in this paper, relax the condition. We do not impose the repeatability on the measurement process, instead we treat the distinguishability of the final states also on the system. We ask for the quantitative trade-off between the distinguishability of the final states on the measured system and the one on the measuring apparatus. According to our result, there is no interaction that achieves perfect distinguishability on both systems. Since our result is quantitative, it enables us to discuss the dependence on the size of the apparatus and the environment.

Let us consider two quantum systems, a system and an apparatus. Each system is described by a Hilbert space, $\mathcal{H}_{S}$ and $\mathcal{H}_{A}$, respectively. Suppose that there exists an additive conservative quantity. That is, there exist an observable $L_{S}$ on the system and an observable $L_{A}$ on the apparatus such that their summation $L_{S}+L_{A}$ is conserved by any physical dynamics for the closed system. Let us consider a pair of orthogonal vector states, $\left|\psi_{0}\right\rangle,\left|\psi_{1}\right\rangle \in \mathcal{H}_{S}$. The goal of the measurement process is to make them distinguishable on the apparatus by choosing an initial state of the apparatus and the interaction between the system and the apparatus. In the case of the ideal measurement, the repeatability of the measurements is also imposed. That is, the states $\left|\psi_{0}\right\rangle$ and $\left|\psi_{1}\right\rangle$ should be invariant with the interaction. We, in this paper, do not employ this repeatability condition. We relax the condition to the distinguishability condition on the system. That is, we ask if it is possible for the final states to be distinguishable on both systems. The distinguishability is characterized by a quantity called fidelity. The fidelity $[8,9]$ between two states $\rho_{0}$ and $\rho_{1}$ is defined by $F\left(\rho_{0}, \rho_{1}\right):=\operatorname{tr}\left(\sqrt{\rho_{0}^{1 / 2} \rho_{1} \rho_{0}^{1 / 2}}\right)$. It takes a nonnegative value less than 1 , and becomes smaller if the states are more distinguishable. The perfect distinguishability corresponds to the vanishing fidelity. The following lemma [10] justifies that the fidelity indeed represents the distinguishability.

Lemma 1 The fidelity equals the square root of minimum overlap coefficient between two probability distributions $p_{0}$ and $p_{1}$ :

$$
F\left(\rho_{0}, \rho_{1}\right)=\min _{\left\{E_{\alpha}\right\}: P O V M} \sum_{\alpha} \sqrt{p_{0}(\alpha) p_{1}(\alpha)},
$$

where $p_{0}$ and $p_{1}$ are defined by $p_{0}(\alpha)=\operatorname{tr}\left(\rho_{0} E_{\alpha}\right)$ and $p_{1}(\alpha)=\operatorname{tr}\left(\rho_{1} E_{\alpha}\right)$. The minimum is taken over all the possible positive operator valued measures (POVMs), where a POVM $\left\{E_{\alpha}\right\}$ is a family of the positive operators satisfying $\sum_{\alpha} E_{\alpha}=1$. Moreover, the minimum is attained by a projection valued measure (PVM), where a PVM $\left\{E_{\alpha}\right\}$ is a family of the projection operators satisfying $\sum_{\alpha} E_{\alpha}=\mathbf{1}$.

This lemma plays an essential role in the proof of our theorem. In the presence of the additive conserved quantity, we have the following theorem. 
Theorem 2 As described above, let us consider a pair of orthogonal states $\left|\psi_{0}\right\rangle,\left|\psi_{1}\right\rangle \in \mathcal{H}_{S}$ in the presence of the additive conserved quantity, $L_{S}+L_{A}$. For any initial state $\sigma$ on the apparatus and the unitary dynamics $U$ satisfying conservation law, the final states $\rho_{0}:=U\left(\left|\psi_{0}\right\rangle\left\langle\psi_{0}\right| \otimes \sigma\right) U^{*}$ and $\rho_{1}:=U\left(\left|\psi_{1}\right\rangle\left\langle\psi_{1}\right| \otimes \sigma\right) U^{*}$ satisfy the following:

$$
\left|\left\langle\psi_{0}\left|L_{S}\right| \psi_{1}\right\rangle\right| \leq\left\|L_{A}\right\| F\left(\rho_{0}^{S}, \rho_{1}^{S}\right)+\left\|L_{S}\right\| F\left(\rho_{0}^{A}, \rho_{1}^{A}\right),
$$

where $\rho_{i}^{S}$ is the final state $\rho_{i}$ restricted to the system and $\rho_{i}^{A}$ is the one restricted to the apparatus, and $F(\cdot, \cdot)$ is the fidelity, and $\|\cdot\|$ represents the operator norm defined as $\|A\|:=\sup _{\varphi \neq 0, \varphi \in \mathcal{H}} \|$ A $\|\varphi\|$ for any operator $A$ on a Hilbert space $\mathcal{H}$.

Proof: By the purification of $\sigma$, we obtain a dilated Hilbert space and a vector state for the apparatus. We write the dilated Hilbert space as $\mathcal{H}_{A}$ for simplicity and the vector state as $|\Omega\rangle$. The dilated unitary operator $U \otimes \mathbf{1}$ is also abbreviated as $U$. Let us define the initial vector states $\left|\Psi_{i}\right\rangle:=\left|\psi_{i}\right\rangle \otimes|\Omega\rangle$ for $i=0,1$. As Wigner-Araki-Yanase's original discussion, we consider the following quantity:

$$
\begin{aligned}
\left\langle\psi_{0}\left|L_{S}\right| \psi_{1}\right\rangle & =\left\langle\Psi_{0}\left|L_{S}+L_{A}\right| \Psi_{1}\right\rangle \\
& =\left\langle\Psi_{0}\left|U^{*}\left(L_{S}+L_{A}\right) U\right| \Psi_{1}\right\rangle \\
& =\left\langle\Psi_{0}\left|U^{*} L_{S} U\right| \Psi_{1}\right\rangle+\left\langle\Psi_{0}\left|U^{*} L_{A} U\right| \Psi_{1}\right\rangle,
\end{aligned}
$$

where in the first line we have used $\left\langle\Psi_{0}\left|L_{A}\right| \Psi_{1}\right\rangle=\left\langle\psi_{0} \mid \psi_{1}\right\rangle\left\langle\Omega\left|L_{A}\right| \Omega\right\rangle=0$. Now we consider an arbitrary projection valued measure $(\mathrm{PVM})\left\{E_{\alpha}\right\}$ on the system and an arbitrary PVM $\left\{P_{j}\right\}$ on the apparatus. Since $\sum_{\alpha} E_{\alpha}=\sum_{j} P_{j}=$ 1 holds, the right hand side of (2) can be written as $\sum_{j}\left\langle\Psi_{0}\left|U^{*} P_{j} L_{S} U\right| \Psi_{1}\right\rangle+\sum_{\alpha}\left\langle\Psi_{0}\left|U^{*} E_{\alpha} L_{A} U\right| \Psi_{1}\right\rangle$. By using commutativity $\left[P_{j}, L_{S}\right]=\left[E_{\alpha}, L_{A}\right]=0$, we obtain,

$$
\left\langle\psi_{0}\left|L_{S}\right| \psi_{1}\right\rangle=\sum_{j}\left\langle\Psi_{0}\left|U^{*} P_{j} L_{S} P_{j} U\right| \Psi_{1}\right\rangle+\sum_{\alpha}\left\langle\Psi_{0}\left|U^{*} E_{\alpha} L_{A} E_{\alpha} U\right| \Psi_{1}\right\rangle
$$

Taking absolute value of the both sides, we obtain,

$$
\begin{aligned}
\left|\left\langle\psi_{0}\left|L_{S}\right| \psi_{1}\right\rangle\right| & \leq \sum_{j}\left|\left\langle\Psi_{0}\left|U^{*} P_{j} L_{S} P_{j} U\right| \Psi_{1}\right\rangle\right|+\sum_{\alpha}\left|\left\langle\Psi_{0}\left|U^{*} E_{\alpha} L_{A} E_{\alpha} U\right| \Psi_{1}\right\rangle\right| \\
& \leq\left\|L_{S}\right\| \sum_{j} \sqrt{\left\langle\Psi_{0}\left|U^{*} P_{j} U\right| \Psi_{0}\right\rangle\left\langle\Psi_{1}\left|U^{*} P_{j} U\right| \Psi_{1}\right\rangle} \\
& +\left\|L_{A}\right\| \sum_{\alpha} \sqrt{\left\langle\Psi_{0}\left|U^{*} E_{\alpha} U\right| \Psi_{0}\right\rangle\left\langle\Psi_{1}\left|U^{*} E_{\alpha} U\right| \Psi_{1}\right\rangle}
\end{aligned}
$$

We here choose the particular PVMs, $\left\{E_{\alpha}\right\}$ and $\left\{P_{j}\right\}$, which attain the fidelity. Thanks to the lemma1, we finally obtain,

$$
\left|\left\langle\psi_{0}\left|L_{S}\right| \psi_{1}\right\rangle\right| \leq\left\|L_{A}\right\| F\left(\rho_{0}^{S}, \rho_{1}^{S}\right)+\left\|L_{S}\right\| F\left(\rho_{0}^{A}, \rho_{1}^{A}\right) .
$$

It ends the proof.

According to this theorem, we obtain the following theorem.

Theorem 3 Under the setting of the theorem2, the perfect distinguishability for both systems cannot be attained simultaneously.

\section{Proof:}

The vanishing fidelities in (1) contradict with the nonvanishing left hand side.

Q.E.D.

Let us consider the simplest example. The system is a spin $1 / 2$ system. The conserved quantity is the $z$-component of the spin, $S_{z}+L_{A}$, where $L_{A}$ is the $z$-component of the spin on the apparatus. $S_{z}$ is written with the eigenvectors, $|1\rangle$ and $|-1\rangle$, as $S_{z}=\frac{\hbar}{2}(|1\rangle\langle 1|-|-1\rangle\langle-1|)$. The observable to be measured $S_{k}$ is a component of spin in another direction. That is, the states to be distinguished by the measurement process are $\left|\psi_{1}\right\rangle:=\alpha|1\rangle+\beta|-1\rangle$ and $\left|\psi_{0}\right\rangle:=\bar{\beta}|1\rangle-\bar{\alpha}|-1\rangle$, where $|\alpha|^{2}+|\beta|^{2}=1$ with $\alpha \neq 0, \beta \neq 0$. The observables $S_{z}$ and $S_{k}$ do not commute with each other. In fact,

$$
\left\langle\psi_{0}\left|S_{z}\right| \psi_{1}\right\rangle=\hbar \alpha \beta
$$

holds. If we assume the rigorous repeatability as in the original Wigner-Araki-Yanase theorem, the state change for the dilated Hilbert space should be written as, 


$$
\left|\psi_{j}\right\rangle \otimes|\Omega\rangle \mapsto\left|\psi_{j}\right\rangle \otimes\left|\phi_{j}\right\rangle
$$

for $j=0,1$. It gives,

$$
\left\langle\psi_{0}\left|S_{z}\right| \psi_{1}\right\rangle=\left\langle\psi_{0}\left|S_{z}\right| \psi_{1}\right\rangle\left\langle\phi_{0} \mid \phi_{1}\right\rangle
$$

and thus $\left|\phi_{0}\right\rangle=\left|\phi_{1}\right\rangle$ holds. Therefore there is no distinguishability on the apparatus side in this case. On the other hand, if we do not impose the repeatability, the distinguishability on both systems is partially attained. In particular, even the perfect distinguishability on the apparatus allows the partial distinguishability on the system. Ohira and Pearle [5] has constructed the following interaction between the system and the spin $1 / 2$ apparatus:

$$
\begin{aligned}
& \left|\psi_{1}\right\rangle \otimes \sqrt{\frac{1}{2}}(|1\rangle+|-1\rangle) \mapsto(\alpha|1\rangle+\beta|-1\rangle) \otimes \sqrt{\frac{1}{2}}(|1\rangle+|-1\rangle) \\
& \left|\psi_{0}\right\rangle \otimes \sqrt{\frac{1}{2}}(|1\rangle+|-1\rangle) \mapsto(\bar{\beta}|1\rangle+\bar{\alpha}|-1\rangle) \otimes \sqrt{\frac{1}{2}}(|1\rangle-|-1\rangle) .
\end{aligned}
$$

It gives the fidelity $F\left(\rho_{0}^{A}, \rho_{1}^{A}\right)=0$ and $F\left(\rho_{0}^{S}, \rho_{1}^{S}\right)=2|\alpha \beta|$. Since $\left\|L_{A}\right\|=\hbar / 2$ holds, this interaction satisfies,

$$
\left|\left\langle\psi_{0}\left|S_{z}\right| \psi_{1}\right\rangle\right|=\left\|L_{A}\right\| F\left(\rho_{0}^{S}, \rho_{1}^{S}\right),
$$

which is the equality version of our theorem.

In the following we consider the effect of the environment. We treat a tripartite system which consists of the system, the apparatus, and the environment. The Hilbert space of the environment is written as $\mathcal{H}_{E}$. On the environment an operator $L_{E}$ is defined, and the conserved quantity is $L_{S}+L_{A}+L_{E}$. We divide the whole system into $\mathcal{H}_{S}$ and $\mathcal{H}_{A} \otimes \mathcal{H}_{E}$. Application of the theorem2 to it derives,

$$
\left|\left\langle\psi_{0}\left|L_{S}\right| \psi_{1}\right\rangle\right| \leq\left(\left\|L_{A}\right\|+\left\|L_{E}\right\|\right) F\left(\rho_{0}^{S}, \rho_{1}^{S}\right)+\left\|L_{S}\right\| F\left(\rho_{0}^{A E}, \rho_{1}^{A E}\right),
$$

where $\rho_{j}^{A E}$ is a state over the apparatus and the environment. Since the partial trace does not reduce the fidelity [10], we obtain,

$$
\left|\left\langle\psi_{0}\left|L_{S}\right| \psi_{1}\right\rangle\right| \leq\left(\left\|L_{A}\right\|+\left\|L_{E}\right\|\right) F\left(\rho_{0}^{S}, \rho_{1}^{S}\right)+\left\|L_{S}\right\| F\left(\rho_{0}^{A}, \rho_{1}^{A}\right) .
$$

This inequality shows that to attain high distinguishability on both systems simultaneously the large apparatus or environment is necessary.

Acknowledgments: T.M. thanks H.Moriya for valuable comments.

[1] E. P. Wigner, Z.Phys. 131,101 (1952).

[2] H. Araki and M. M. Yanase, Phys.Rev.120,622 (1960).

[3] M. M. Yanase, Phys.Rev.123,666 (1961).

[4] G. C. Ghirardi, F. Miglietta, A. Rimini, T.Weber, Phys.Rev.D 24, 347,353 (1981).

[5] T. Ohira, P. Pearle, Am.J.Phys.56(8), 692 (1988).

[6] M. Ozawa, Phys. Rev. Lett. 88, 050402 (2002).

[7] M. Ozawa, in Proceedings of the Sixth International Conference on Quantum Communication, Measurement and Computing, edited by J. H. Shapiro and O. Hirota (Rinton Press, Princeton, 2003), pp. 175-180.

[8] A. Uhlmann, Rep.Math.Phys. 9,273 (1976).

[9] R. Jozsa, J.Mod.Opt. 41, 2315 (1994).

[10] H. Barnum, C. M. Caves, C. A. Fuchs, R. Jozsa, B. Schumacher, Phys.Rev.Lett.76 2818 (1996). 